\title{
DESIGN AND IN VITRO CHARACTERIZATION OF METFORMIN LOADED RESEALED ERYTHROCYTES
}

\author{
AISWARYA S, PARVATHY S, ANEESH TP*, VIDYA VISWANAD* \\ Amrita School of Pharmacy, Amrita University, Amrita Vishwa Vidyapeetham, AIMS Health Sciences Campus, Kochi, Kerala, India. Email: \\ vidyaviswanad@aims.amrita.edu/aneeshtp@aims.amrita.edu
}

Received: 15 December 2016, Revised and Accepted: 16 January 2017

\section{ABSTRACT}

Objective: Metformin is an oral antidiabetic drug in the biguanide class. It is the first-line drug of choice for the treatment of type 2 diabetes. The objective of this study was to retard the release of metformin using carrier erythrocyte for getting a parenteral slow release depot formulation.

Methods: The study retards the release of metformin by encapsulating in carrier erythrocyte. Endocytosis is the method used for the encapsulation of the drug metformin.

Results: The optimized formulation shows $98.34 \%$ of drug release within 12 days. From the in vitro release data, zero order kinetic graph shows the best fit graph. \% cell recovered was found to be $73-78 \%$ and it suggests that the loading technique was less destructive.

Conclusion: The optimized formulation is a perfect carrier for the parenteral slow release depot of metformin.

Keywords: Resealed erythrocytes, Cross-linking, Cell recovery, Endocytosis, Slow release depot formulation.

(C) 2017 The Authors. Published by Innovare Academic Sciences Pvt Ltd. This is an open access article under the CC BY license (http://creativecommons. org/licenses/by/4. 0/) DOI: http://dx.doi.org/10.22159/ajpcr.2017.v10i4.16636

\section{INTRODUCTION}

Diabetes mellitus is an aggregation of metabolic illness with high blood glucose levels that result from defects clinched alongside insulin response secretion, alternately action, or both. It's a disease that can be classified under the category of chronic systemic diseases (CSDs). CSD are diseases that affect the whole body or different part of the body [1]. CSD diabetes mellitus is an aggregation of metabolic illnesses portrayed eventually Tom's perusing helter skelter blood glucose levels that result from defects clinched alongside insulin response secretion, alternately action, or both. Most often CSDs are treated with oral drugs. Some of the drugs used for these chronic diseases include drugs having relatively shorter half-life. Hence, a multiple dosage regimen is needed to maintain the therapeutic plasma level concentration which leads to toxicity due to the accumulation [2]. Biguanide drug metformin is used in the treatment of type 2 diabetes mellitus and polycystic ovary syndrome. It is available in market as oral tablet. Administration of the drug by oral route is often associated with several gastrointestinal problems. The bioavailability of the drug is also low due to poor intestinal permeability. Therefore increased the dosage of metformin is usually advised to the patients to obtain desired therapeutic concentration. Furthermore, the shorter half-life of the drug in systemic circulation increases the frequency of its administration [3]. Developing a parenteral controlled slow release formulation is, therefore, essential so that it can bypass the gastrointestinal side effects and reduces the frequency of administration of metformin. Incorporation of the drug in vesicular bodies is a usually adopted strategy for parenteral slow release formulation but it possesses a disadvantage of rapid clearance from circulation by Reticuloendothelial system (RES) [4]. So incorporating body's own erythrocyte may avoid RES clearance. Erythrocytes are made of cell membrane which constitutes phospholipid bilayer like any novel vesicular bodies [5,6]. However, they are not recognized by the macrophage system because of variety of proteins residing on the red blood cell (RBC) membrane. After all, change in membrane structure occurs and these cells are taken up by RES and are destroyed. Then, either the drug is cleared by the system or it is further reached the systemic circulation by the process known as extravasation. Hence, the drug is continuously supplied for a prolonged basis [7]. Phospholipids in erythrocytes are found as bilayer with choline-containing phospholipidlike phosphatidylcholine (PC) and sphingomyelin (SM) in the outer layer and amine containing phospholipid-like phosphatidylethanolamine and phosphatidylserine (PS) in the inner layer. Erythrocytes can be used as drug carrier for slow release depot formulation provided that there should not any exposure of PS during the loading procedure. Exposure of PS is preferred if we are supposed to target RES system because PS is a self-marker for macrophages. There are various methods for loading drug in erythrocytes. However, all the methods are not equally suitable for loading drugs to treat CSDs. The method we adopt should not cause any structural change during the treatment. This can be confirmed by viewing under an electron microscope. Any plasma membrane deformity occurs during the loading processes may cause it recognized by the macrophages, and they will be cleared off from the circulation. Endocytosis is used for loading metformin in erythrocyte as it is a less destructive method. Endocytosis occurs by engulfing the substance into the erythrocytes similar to phagocytosis [8]. Substance to be entrapped into the RBC should have a degree of water solvability and resistivity to a basement within the erythrocytes [9]. As endocytosis is a less destructive method, greater circulation half-life is achieved. Type 2 diabetes mellitus and polycystic ovary syndrome are chronic diseases requiring long-term therapy, the long circulation half-life and prolonged zero order release are usually preferable. When this condition is achieved, the dose and frequency of administration of metformin can be reduced. Delivering metformin loaded erythrocytes intravenously will probably reduce gastrointestinal side effects by bypassing the gastrointestinal route. Reduction in dosing frequency and controlled release of metformin will reduce the dose-related side effect like lactic acidosis. Hence, the object of our present study is to imbecile the release of metformin using carrier erythrocyte for getting a parenteral slow release depot formulation.

\section{METHODS}

Metformin which is obtained as gift sample from Yarrow Chem, Mumbai, adenosine triphosphate is from Loba Chemie Pvt. Ltd, Mumbai, Calcium 
chloride is obtained from Nice Chemicals, Kochi. Moreover, all other chemicals used in this study are of analytical reagent grade.

\section{Physical interaction of RBC with metformin}

Fourier transform infrared (FTIR) spectra

FTIR spectra of metformin, RBC, and metformin-RBC are taken and studied for the interaction. Blood samples were lyophilized, and FTIR was taken.

\section{In silico analysis}

Docking studies were carried out using Auto dock 4.2 between human oxy hemoglobin chain A (PDB: 2DN1) and metformin after identification of the possible binding pocket using CASTp. AutoDock uses one of the several conformational search algorithms to explore the conformational states of a flexible ligand, using the maps generated by AutoGrid to evaluate the ligand-protein interaction at each point in the docking simulation. Here, in this study, metformin-human oxy hemoglobin interaction was studied.

\section{Collection of erythrocytes}

RBC was collected from the blood bank of Amrita Institute of Medical Sciences, Kochi. The institution has an informed consent form from donors that their blood can be used for biomedical research. RBC was washed with phosphate buffered saline (PBS) $(\mathrm{pH}$ 7.4) to remove the traces of heparin because it acts as an analytical interference during the study as the absorption maxima of heparin and metformin is $232 \mathrm{~nm}$. The supernatant buffer was removed by aspiration followed by centrifugation to get packed volume cell. Instead of distilled water for washing erythrocyte care was taken for using PBS 7.4 because distilled water is a perfect hemolysing agent.

\section{Loading of metformin in RBC}

For loading metformin into erythrocyte, concentrations having no or little hemolysis were selected. For that, a series of the concentration of metformin ranging from 1 to $10 \mathrm{mg} / \mathrm{ml}$ was prepared. The solutions were then added to RBC suspended in PBS and allowed to stand for an hour. The supernatant liquid was withdrawn, and \% hemolysis was determined for each concentration of metformin. The percentage hemolysis was calculated using the formula.

$$
\% \text { hemolysis }=\frac{\text { Absorbance of sample }- \text { absorbance of blank }}{\text { Absorbance of positive control }} \times 100
$$

The supernatant obtained by adding triton $\mathrm{X}$ to erythrocyte suspension was used as positive control. $1 \% \mathrm{v} / \mathrm{v}$ of triton X is a detergent with $100 \%$ hemolyzing capacity. The supernatant obtained when adding normal saline to the erythrocyte suspension was used as blank. The absorbance was ultraviolet (UV) spectrophotometrically determined at $540 \mathrm{~nm}$. By determining the $\%$ hemolysis, we can optimize the concentration of metformin to be loaded to erythrocytes. Drug loading was done by the method of endocytosis and normal transportive mechanism.

\section{Method of endocytosis}

To $5 \mathrm{ml}$ of buffer containing $2.5 \mathrm{mmol}$ ATP, $1 \mathrm{mmol} \mathrm{CaCl} \cdot 2 \mathrm{H}_{2} \mathrm{O}$ and $2.5 \mathrm{mmol} \mathrm{MgCl}_{2}, 1 \mathrm{ml}$ of selected concentrations of metformin were added. To the solution, $0.65 \mathrm{ml}$ of erythrocyte was added and incubated at $0^{\circ} \mathrm{C}$ for 15 minutes. Then, it was resealed using $0.9 \%$ sodium chloride solution at $37^{\circ} \mathrm{C}$. The suspension then obtained was washed twice with cold PBS followed by centrifugation at $5000 \mathrm{rpm}$ for 15 minutes [10]. The method of endocytosis versus normal transportive mechanism at our different mechanism was shown in Table 1.

\section{Normal transportive mechanism}

To $5 \mathrm{ml}$ of PBS 7.4, $1 \mathrm{ml}$ of selected concentrations of metformin was added. To the solution, $0.65 \mathrm{ml}$ of erythrocyte was added and incubated at $0^{\circ} \mathrm{C}$ for 15 minutes. Then, it was resealed using $0.9 \%$ sodium chloride solution at $37^{\circ} \mathrm{C}$. The suspension then obtained was washed twice with cold PBS followed by centrifugation at $5000 \mathrm{rpm}$ for 15 minutes. The centrifugation was done to remove the metformin that was bound to the surface of RBC membrane [11].

Summary of $2 * 4$ factorial ANOVA and post-hoc Tuckey HSD test was shown in Tables 2 and 3.

The loaded erythrocytes were further in vitro characterized for their suitability as slow release parenteral depot.

\section{Characterization of metformin loaded resealed erythrocytes}

The in vivo performance operation of resealed erythrocyte is affected to a great extent by their in vitro biological prop of erythrocytes. Hence, in vitro characterization $\mathrm{f}$ Mannikin an important part of the study involving the use of such cellular carriers.

\section{Encapsulation efficiency}

The encapsulation efficiency was calculated for erythrocytes loaded by endocytosis and was compared with drug loaded by normal transportive mechanism. In normal transportive mechanism, the drug loading was carried out in PBS alone. This method involves the loading of erythrocytes with drug without disrupting the erythrocyte membrane in by incubating the drug and erythrocytes. To determine encapsulation efficiency, the RBC membrane was deproteinized using methanol followed by centrifugation. $1 \mathrm{ml}$ of the supernatant was pipetted out and drug content was determined using UV-visible spectrophotometer at $232 \mathrm{~nm}$ and encapsulation efficiency was determined.

Encapsulation efficiency $=\frac{\text { Encapsulated drug }}{\text { Total drug }} \times 100$

Table 1: Data table of endocytosis method versus normal transportive mechanism at four different concentrations

\begin{tabular}{lllll}
\hline Method & Concentration $\mathbf{1 ~} \mathbf{~ g g}$ & Concentration $\mathbf{4} \mathbf{~ m g}$ & Concentration $\mathbf{7 ~ m g}$ & Concentration 10 mg \\
\hline Normal transportive mechanism & 123.4 & 1595.20 & 4223.80 & 6988 \\
Endocytosis & 310.6 & 1898.4 & 2081.8 & 2046 \\
\hline
\end{tabular}

Table 2: $2 \times 4$ factorial ANOVA table for the influence of method and concentrations

\begin{tabular}{llllll}
\hline ANOVA summary & & & & \\
\hline Source & SS & df & MS & F & p \\
\hline Method of loading $(\mathrm{r})$ & 19559436.87 & 1 & 19559436.87 & 4602.31 & $<0.0001$ \\
Concentration added (c) & 66964180.83 & 3 & 22321393.61 & 5252.2 & $<0.0001$ \\
r $\times$ c & 24132854.5 & 3 & 8044284.83 & 1892.81 & $<0.0001$ \\
Error & 72248.56 & 17 & 4249.92 & & \\
Total & 110728720.76 & 24 & & & \\
\hline
\end{tabular}

ANOVA: Analysis of variance 
In vitro drug release

The drug release study for the concentration with highest loading efficiency was carried out for 12 days in an orbital shaking incubator at $37^{\circ} \mathrm{C} .1 \mathrm{ml}$ of the supernatant layer was withdrawn at various time intervals. It was then deproteinized using methanol and passed through $0.22 \mu \mathrm{m}$ syringe filter to remove the precipitated protein. The amount of metformin released at each time interval was estimated by UV spectrophotometry at $232 \mathrm{~nm}$. To study the release kinetics, the data obtained from in vitro drug release studies were plotted in various kinetic models [12].

\section{Effect of cross-linking agent on drug release}

The loaded erythrocytes were treated with glutaraldehyde crosslinker. In vitro drug release was also taken for glutaraldehyde treated formulations to study the effect of concentration of cross-linking agent on drug release. The concentration of cross-linking agent was varied from $0.2-0.8 \% \mathrm{v} / \mathrm{v}$ and was treated to erythrocytes loaded with optimized concentration. Glutaraldehyde was used as cross-linking agent, as it makes more chemically and thermostable cross-links with protein than other aldehyde crosslinkers [13].

Hematological parameters, such as osmotic fragility test and turbulence shock, were studied and compared with normal erythrocytes.

\section{Osmotic fragility test}

Osmotic fragility test was carried out for both loaded and unloaded erythrocytes. For this, a series of concentration of sodium chloride ranging from $0.1 \%$ to $0.9 \% \mathrm{w} / \mathrm{v}$ was prepared. A drop of the loaded and unloaded erythrocytes was added separately to different concentrations of sodium chloride taken in test tube. Then, it was centrifuged and allowed to stand for $1 \mathrm{hr}$ at $37^{\circ} \mathrm{C}$. The supernatant was taken, and the absorbance was noted at $540 \mathrm{~nm}$ in a UV-visible spectrophotometer [14]. Osmotic fragility curve was then constructed by plotting concentration of sodium chloride in X axis and for both loaded and normal erythrocyte. Statistical analysis was applied to predict the significant difference between the loaded and normal erythrocytes.

\section{Turbulence shock studies}

Turbulence stupor enables an evaluation to be made of the stability of the loaded erythrocytes against the Sturm und Drang accent exerted by the cells against in-vivo circulation turbulence. The trial run is performed by the method of Deloach et al. whereby the erythrocyte suspension was passed through a 22-standard of measurement needle several times at a flow rate of $10 \mathrm{ml} /$ minutes and \% hemolysis was calculated. The number of passes was plotted against \% hemolysis for both loaded and unloaded erythrocytes [15].

\section{Cell counting and cell recovery}

This involves count the identification number of bolshie ancestry cells per unit of measurement intensity of whole blood, usually by automated counting. Red cell recovery may be calculated on the basis of the differences in the hematocrit and the volume of the suspension of erythrocytes before and after loading. The goal is to minimize the loss during the encapsulation subprogram to maximize cell coalescence.

Cell recovery means a number of cells retained after loading. Percent cell recovery was determined by counting the number of intact cells per cubic $\mathrm{mm}$ of packed erythrocytes before and after loading the drug.

\section{Number of cells before loading \\ $\%$ cell recovery $=\frac{- \text { Number of cells after loading }}{\text { Number of cells before loading }} \times 100$}

\section{Morphological studies}

JEOL JSM-6390 LA scanning electron microscope (Jeol Ltd., Tokyo, Nippon) prepared with a digital camera, at $20 \mathrm{kV}$ speeding up voltage was used to (figure out the worth, amount, or quality of) the (related to shape and structure) differences between (usual/commonly and regular/healthy) and metformin loaded erythrocytes. After the samples
Table 3: Post-hoc Tukey HSD test

\begin{tabular}{lll}
\hline \multicolumn{3}{l}{ Critical values for the Tukey HSD test } \\
\hline Parameters & HSD (0.05) & HSD (0.01) \\
\hline Rows (2) & 54.98 & 75.65 \\
Columns (4) & 105.1 & 134.48 \\
Cells (8) & 179.83 & 222.98 \\
\hline
\end{tabular}

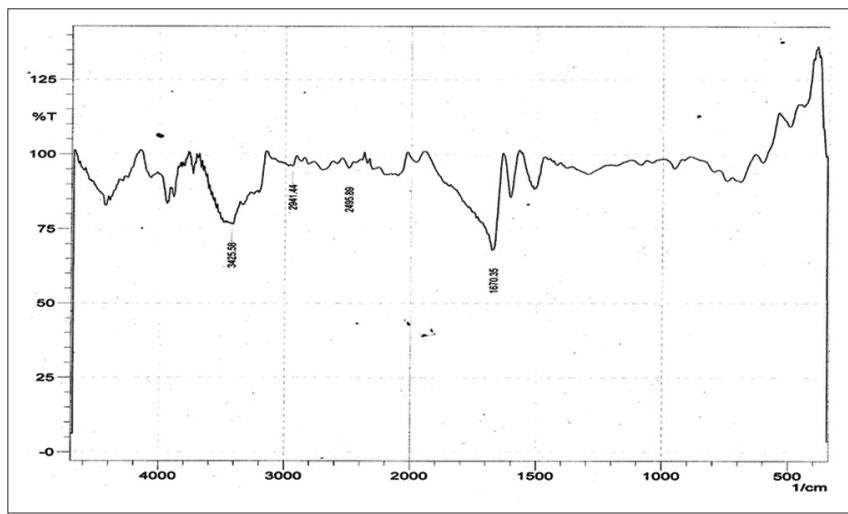

Fig. 1: Fourier transform infrared of metformin

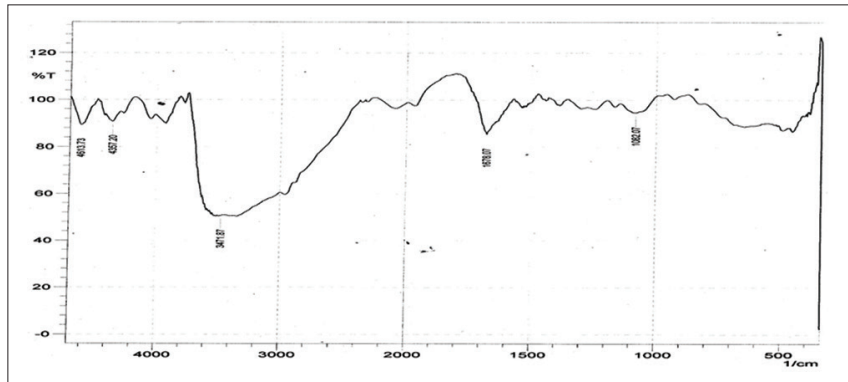

Fig. 2: Fourier transform infrared of red blood cell

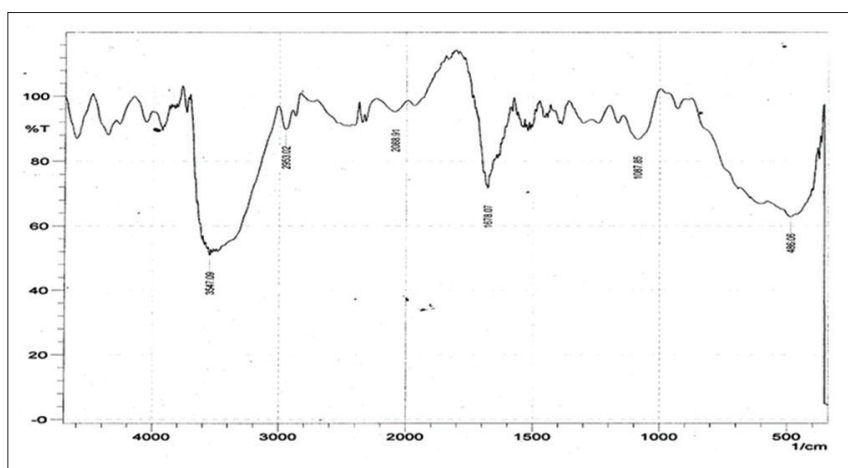

Fig. 3: Fourier transform infrared of red blood cell-metformin

were fixed in buffered glutaraldehyde, the aldehyde medium was drained off. The cells were rinsed 3 times for 5 minutes in phosphate buffer and after fixed in osmium tetroxide for $1 \mathrm{hr}$. The samples were then rinsed with produced/showed water and having no water or not enough water) using a graded ethanol series: $25,50,75,100$, and another $100 \%$, each for 10 minutes. The samples were rinsed in water, removed, mounted on studs, sputter-coated with gold, and then viewed using scanning electron micrograph (SEM) [16].

Determination of phospholipid asymmetry

Phospholipid asymmetry of loaded and unloaded erythrocytes was studied by Annexin V binding assay using flow cytometer. $20 \mu \mathrm{l}$ of RBC 


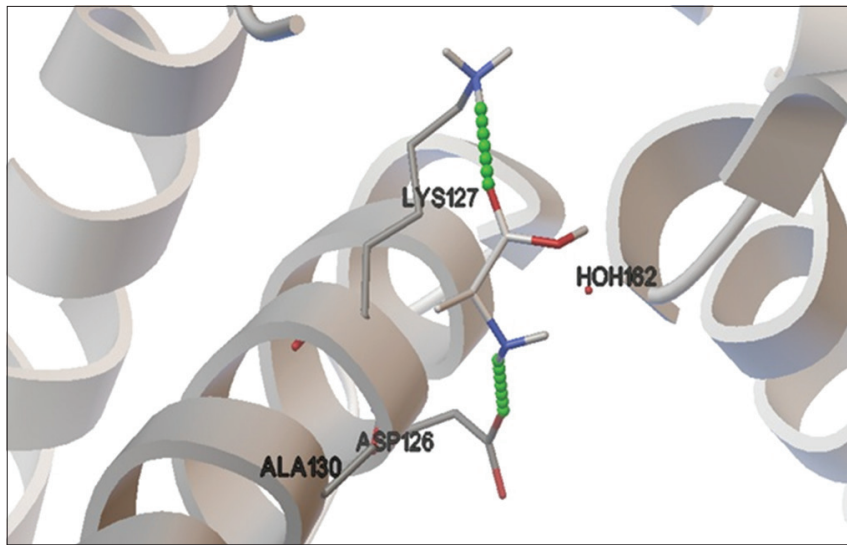

Fig. 4: Docking study result between metformin and hemoglobin

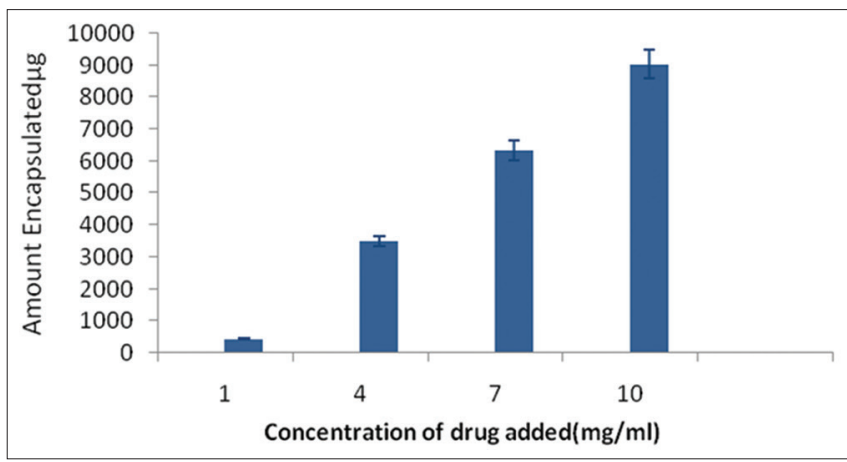

Fig. 5: The amount encapsulated in erythrocyte at 4 different concentrations of metformin by the method of endocytosis

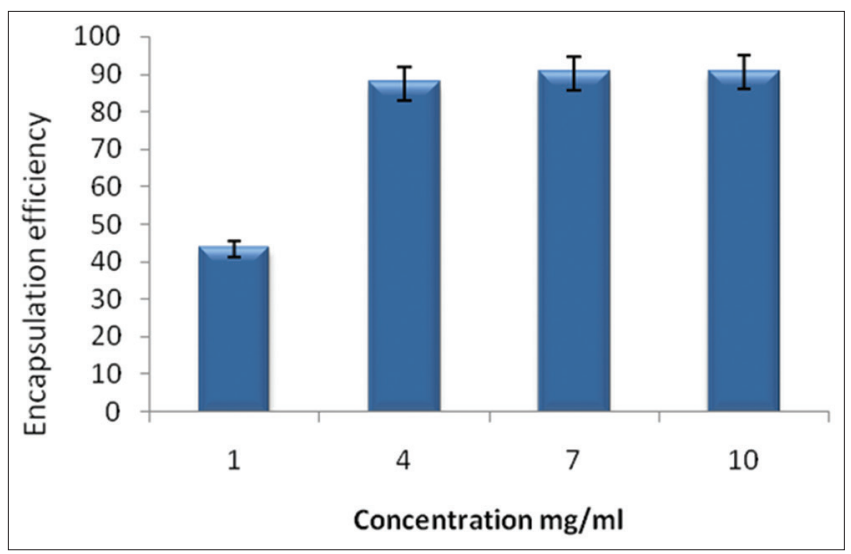

Fig. 6: The encapsulation efficiency at four different concentration by the method of endocytosis

suspension was taken. Annexin $\mathrm{V}$ binding buffer was added to it, and then it is fluorescently labeled with fluorescein isothiocyanate and incubated for 15 minutes at the dark condition and detect the presence of PS exposed cell.

\section{Mean corpuscular volume (MCV)}

MCV is the average volume of red cells in a specimen. Both loaded and normal erythrocytes were made to $50 \%$ hemocrit. For making $50 \%$ hemocrit $0.5 \mathrm{ml}$ of packed volume cell of both loaded and normal erythrocytes were made to $1 \mathrm{ml}$ using normal saline.

$\mathrm{MCV}=\frac{10 \times \text { hematocrit }(\%)}{\mathrm{RBC} \text { count }(\text { millions } / \mu \mathrm{l})}$

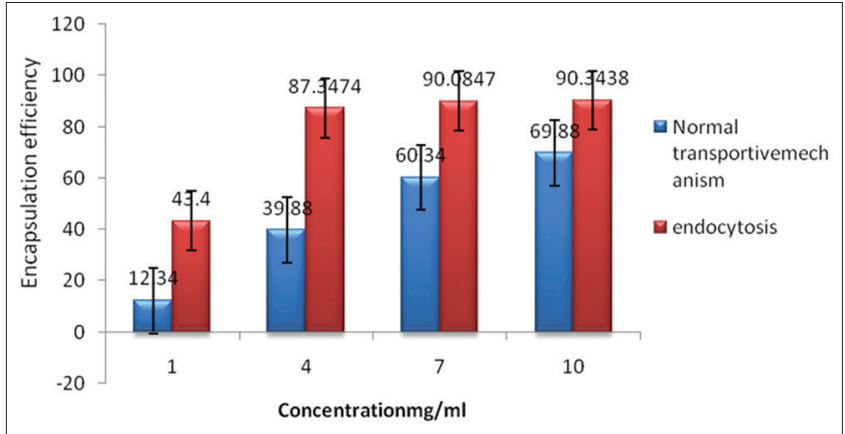

Fig. 7: Comparison of encapsulation efficiency of metformin loaded by endocytosis method with normal transportive mechanism at 4 different concentration. Data are expressed as mean \pm standard deviation, 3 samples in each group $n=3.2 \times 4$. Two-way factorial analysis of variances was carried out followed by post hoc Tukey HSD test. $\mathbf{p}<\mathbf{0 . 0 0 0 1}$ suggests that method of endocytosis had significant role in metformin loading

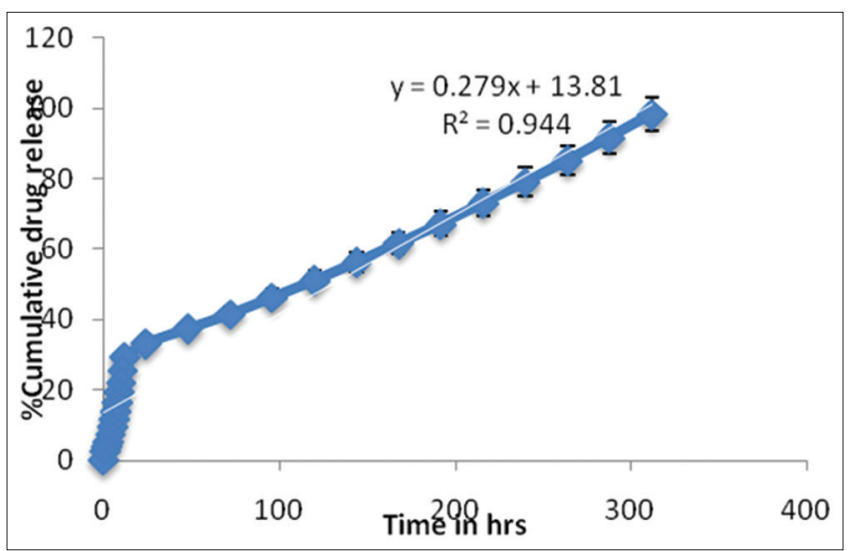

Fig. 8: Percentage cumulative drug release of Fo formulation against time. It take $312 \mathrm{hrs}$ (12 days) to release $98.33 \%$ of drug. Data are expressed as mean \pm standard deviation, 3 samples in each group $n=3$

\section{Stability studies}

No prescribed guidelines are there for the determination of stability of resealed erythrocytes. According to published articles, the resealed erythrocytes have to be stored in lyophilized form. Hence, the prepared resealed erythrocyte formulation was lyophilized. Lyophilized product stability was assessed by determining the $\%$ hemolysis after re-suspending the product [17]

\section{RESULTS AND DISCUSSION}

\section{Physical interaction of RBC with metformin}

The physical interaction of metformin with RBC and metformin with hemoglobin was studied. Physical interaction of metformin with RBC was studied by taking FTIR spectra of metformin, lyophilized RBC and physical mixture of lyophilized RBC and metformin kept for 7 days. The physical interaction of metformin with hemoglobin was studied by docking metformin with human oxy hemoglobin chain A.

\section{FTIR of metformin}

FTIR of metformin showed a strong absorption band at 1672 and 1512 is due to $\mathrm{C}=\mathrm{N}$ stretching vibration. A very weak band in the region of 1220-1020 indicates the presence of aliphatic amine. The band between the regions of 3400 and 3100 is due to $\mathrm{N}-\mathrm{H}$ symmetric and asymmetric stretching vibration. The very weak intensity band at 910660 is due to $\mathrm{N}-\mathrm{H}$ Wagging. In addition, the presence of methyl group is 


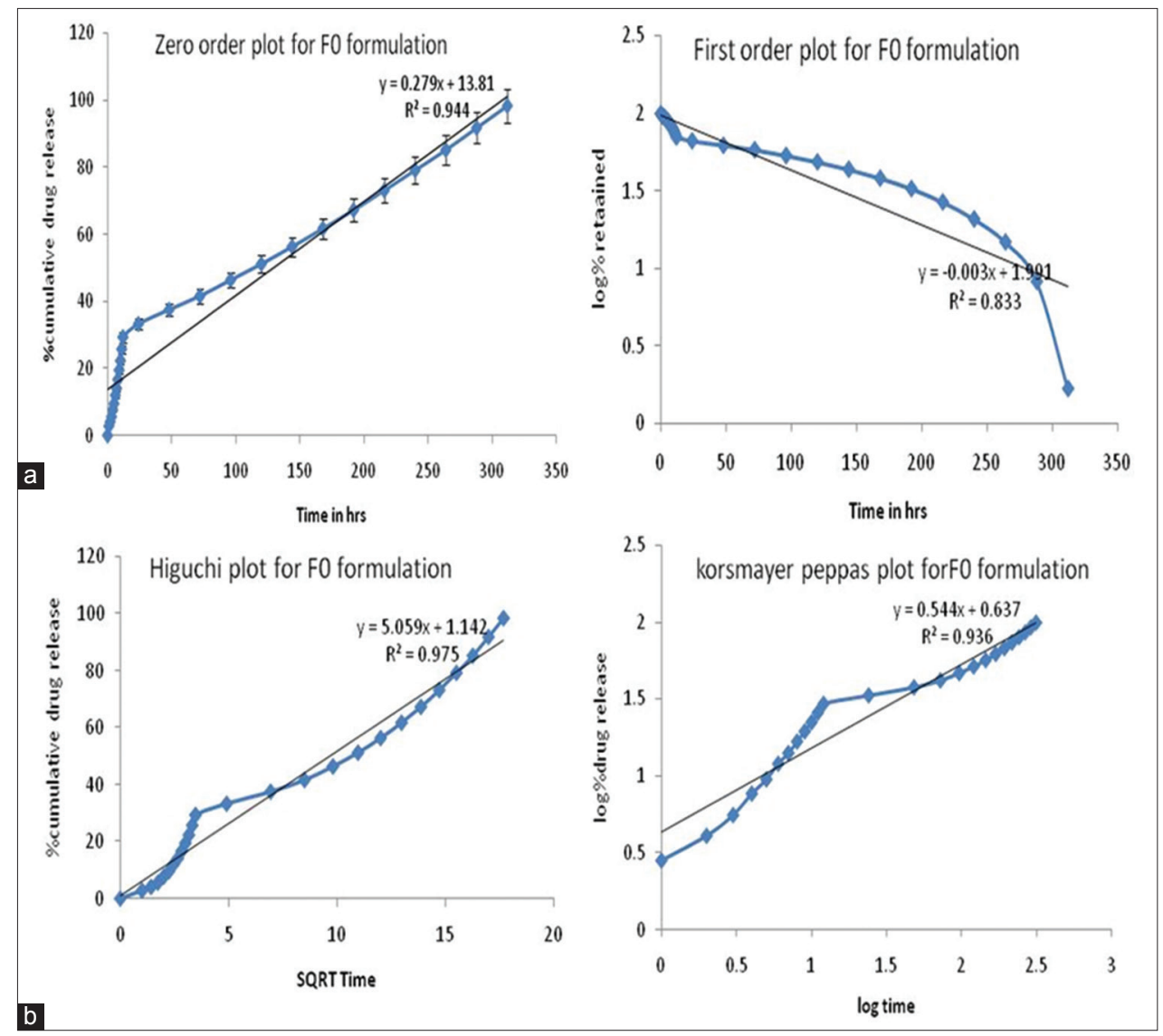

Fig. 9: ( $a$ and $b$ ) Kinetic data modeling for Fo formulation

confirmed by the weak intensity band at 2800 range. FTIR of Metformin is shown in Fig. 1.

\section{$\mathrm{N}-\mathrm{H}$ stretching vibrations}

The N-H stretching vibrations are found in the region $3550-3250 \mathrm{~cm}^{-1}$. In this study, medium to strong intensity band appearing at $3425 \mathrm{~cm}^{-1}$ is assigned to $\mathrm{N}-\mathrm{H}$ stretching vibration. The band appearing at 3176 and $3300 \mathrm{~cm}^{-1}$ are of medium intensity in nature and have been assigned as symmetric and asymmetric $\mathrm{N}-\mathrm{H}$ stretching vibrations, respectively.

\section{$\mathrm{N}$-H deformation vibrations}

The $\mathrm{N}-\mathrm{H}$ deformation vibrations occur in the region of $1650-1581 \mathrm{~cm}^{-1}$. In the present spectra, strong absorption occurs at 1672, 1602, 1512 frequencies.

\section{C-N stretching}

The C-N stretching vibrations generally occur in the region $1170-1040 \mathrm{~cm}^{-1}$. In this study, the weak and medium to weak intensity bands appearing at frequencies 1170, 1097 and $1033 \mathrm{~cm}^{-1}$ in IR spectrum.

\section{$\mathrm{N}-\mathrm{H} 2$ rocking vibrations and $\mathrm{N}-\mathrm{H}$ wagging}

In metformin hydrochloride, a weak band at $800 \mathrm{~cm}^{-1}$ have been assigned to $\mathrm{N}-\mathrm{H} 2$ rocking vibrations. $\mathrm{N}-\mathrm{H}$ wagging vibrations appear at the region $910-660$.

\section{FTIR of RBC}

FTIR of RBC shows a broad peak in 3000-3600 range is peculiar for $\mathrm{OH}$ stretching of the group present in phospholipid sphingomyelin in the outer layer of RBC. One weak band just below 3000 is attributed due to $\mathrm{CH}$ stretching vibration. The peak at 1678 is due to Amide I shift and weak peak below 1500 is due to Amides II and III shift. Symmetric and asymmetric stretching vibrations from the phosphate group are seen in the FTIR spectra in the region of $950-1250 \mathrm{~cm}^{-1}$. FTIR of RBC was shown in Fig. 2.

\section{FTIR of physical mixture of lyophilized RBC and metformin} Prominent Peaks present in metformin is also present in RBC. Hence, when the FTIR spectra were taken for metformin-RBC complex, the addition of peaks occur due to Fermi resonance. The broad peak at 3344 may be due to the interaction of $\mathrm{N}-\mathrm{H} 2$ group with the hydrophilic domain of cell membrane. This is advantageous for the process of endocytosis. For the process of endocytosis to be occurring, the drug molecule should possess a group that binds with the hydrophilic domain of the membrane. Only after binding with the hydrophilic domain the process of endocytosis takes place. FTIR of RBC-Metformin was shown in Fig. 3.

\section{Physical interaction of metformin with hemoglobin}

The docking study reveals the presence of hydrogen bonding between the drug and amino acids ASP126 and LYS127. The binding score was -3.33 , and the absence of other interactions like cation-cation interaction, or pi cation interaction suggests only a weak interaction was there between metformin and hemoglobin. Docking results of Metformin with Hemoglobin was shown in Fig. 4.

\section{Characterization of metformin loaded resealed erythrocytes Encapsulation efficiency}

The encapsulation of metformin in erythrocytes was done by the method of endocytosis and by normal transportive mechanism. The encapsulation efficiency was calculated for erythrocyte loaded by both the method. Concentrations 1, 4, 7, $10 \mathrm{mg} / \mathrm{ml}$ of metformin were used for encapsulation. The amount encapsulated in erythrocytes at 4 different concentrations of Metformin is showed in Fig. 5.

From the graph, it was evident that when concentration $10 \mathrm{mg} / \mathrm{ml}$ was used the amount of metformin encapsulated is greater. 
The corresponding encapsulation efficiency of different concentration of metformin was calculated. The encapsulation efficiency at four different concentration of Metformin was shown in Fig. 6.

The encapsulation efficiency was calculated for erythrocytes loaded by endocytosis and was compared with drug loaded by normal transportive mechanism. In normal transportive mechanism, the drug loading was carried out in PBS alone. To determine encapsulation efficiency, the RBC membrane was deproteinized using methanol followed by centrifugation. $1 \mathrm{ml}$ of the supernatant was pipetted out, and drug content was determined using UV-visible spectrophotometer at $232 \mathrm{~nm}$ and encapsulation efficiency was determined. Comparison of encapsulation efficiency of metformin loaded by endocytosis method with normal transportive mechanism at 4 different concentration is shown in Fig. 7.

To check whether endocytosis has any significant role in metformin loading $2 \times 4$ two-way factorial analysis of variance (ANOVA) was performed. In two-way ANOVA main effect and interaction effect can be calculated. By comparing the method of endocytosis with normal transportive mechanism, it was revealed that the mechanism of drug loading depend not only on endocytosis, some amount of encapsulation occur due to the effect of diffusion also.

The encapsulation efficiency may depend on two factors methods of drug loading and concentration. Thus it has 8 treatment groups. (First factor have 2 level, while second factor have 4 level; $2 \times 4=8$ ). The degree of freedom (DF) for the type of method is $2-1=1$ and $D F$ for concentration is $4-1=3$. There are $1 \times 3=3$ degrees of freedom for the interaction between the type of method and concentration.

The HSD test between row means can be meaningfully performed only if the row effect is significant; between column means, only if the column effect is significant; and between cell means, only if the interaction effect is significant. Thus, it is relevant that method of endocytosis has any significant role in metformin loading.

In normal transportive mechanism, drug enters into the erythrocytes by simple diffusion. The metformin loaded into erythrocytes by the mechanism of diffusion will bind with hemoglobin by forming hydrogen bond (Met---Hb). Metformin loaded into erythrocyte by the process of endocytosis will reside inside the endocytic vacuoles.

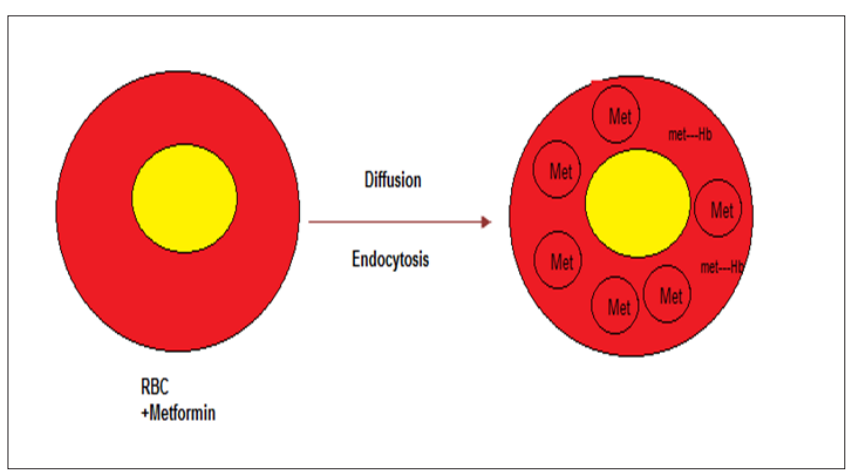

\section{In vitro drug release}

The drug release study for the concentration with highest loading efficiency was carried out and is given below. $10 \mathrm{mg} / \mathrm{ml}$ concentration loaded by the method of endocytosis was having highest loading efficiency and it was used for in vitro drug release study. To further retard the release rate crosslinker was added. Glutaraldehyde was used here as the cross-linking agent, as it makes more chemically and thermostable cross-links with protein than other aldehyde crosslinkers. Glutaraldehyde of concentrations $0.2 \%, 0.4 \%, 0.6 \%$ and $0.8 \% \mathrm{v} / \mathrm{v}$ were added to F0 formulation to get F1, F2, F3 and F4 formulations, respectively. Percentage cumulative drug release of F0 Formulation was shown in Fig. 8.

\section{Effect of cross-linking agent in drug release}

In vitro release of metformin was also taken for glutaraldehyde treated formulation in order to study the effect of concentration of glutaraldehyde on release rate. It was found that as the concentration of cross-linking agent increases release rate found to be decreased. The release data were taken only for 12 days because the in vitro stability of resealed erythrocyte formulation at $37^{\circ} \mathrm{C}$ is less. The percentage cell recovered after 12 days were decreasing to a significant extent and also the formulation is susceptible to contamination. From the graph, it was found that as the concentration of glutaraldehyde increases the release rate found to be decreased. To check the statistical significance of release rate retardation One-way ANOVAs test followed by bonferonni multiple comparison test was carried out and suggest the concentration of glutaraldehyde have a significant role in release retardation. The post hoc test bonferronni multiple comparison tests was meaningfully performed only when there was a significant difference in release rate of formulations at $95 \%$ confidence interval.

\section{Kinetic modeling of release rate}

Like any vesicular bodies, erythrocyte is made up of phospholipid bilayer. Kinetic model is applied to predict the release pattern from loaded erythrocytes in vitro. $98.34 \%$ of drug is released within 12 days from F0 formulation. Therefore, the kinetic model is applied to glutaraldehyde untreated F0 formulation as we can predict model after almost all encapsulate drug is release. Kinetic data modelling for F0 formulation is shown in Fig. 9.

$\mathrm{R}^{2}$ value of zero order kinetics was greater compared to first order kinetics. Hence, the best fit graph is the zero order kinetic graph. In vitro release of metformin from loaded erythrocytes follows zero order kinetics. To confirm this and predict the mechanism of release Higuchi plot and Korsmeyer-Peppas model was constructed. The $\mathrm{n}$ value of Korsmeyer-Peppas graph was $>0.5$ suggests non-Fickian diffusion, i.e., dissolution followed by diffusion. The initial retardation in release rate might be due to the weak interaction between metformin and hemoglobin (the docking energy of -3.33), the hydrogen bonding between the drug and amino acids ASP126 and LYS127. Hence, the breaking of this hydrogen bond is required for the release of metformin from erythrocyte. Further release occurs from endocytic vesicles.

Several hematological parameters were also assessed for loaded and unloaded erythrocyte to predict any significant difference between loaded and unloaded cell.

\section{Osmotic fragility}

It is a test that measures the resistance to blood destruction of red platelets (RBC) presented to hypotonic solutions. RBC are presented to a progression of salt water $(\mathrm{NaCl})$ solutions with increasing dilution. The prior blood destruction happens, the more prominent is osmotic easily-broken quality of RBC. RBC burst in hypotonic $(<0.9 \% \mathrm{NaCl})$, and shrink (crenate) in hypertonic solutions $(>0.9 \%$ $\mathrm{NaCl}$ ). In hypotonic medium a membrane break happens, allowing (a part of the blood that carries oxygen) ( $\mathrm{Hb}$ ) to exit from the cells. By measuring $\mathrm{Hb}$ concentration, the $\%$ of blood destruction at different $\mathrm{NaCl}$ concentrations can be calculated. Normally hemolysis onset at $0.45-0.5 \% \mathrm{NaCl}$ and complete at: $0.3-0.33 \% \mathrm{NaCl}$. Osmotic fragility is affected by cell membrane permeability and surface-to-volume ratio. This test of resealed erythrocytes is an indicator of the possible changes in cell membrane (honest and good human quality/wholeness or completeness) and the resistance of these cells to osmotic pressure of the suspension medium. Osmotic fragility test gives an idea about the fragility of erythrocytes with respect to the change in tonicity of the surrounding of erythrocytes. Osmotic fragility of both loaded and normal erythrocytes are calculated and are given in Fig. 10. The loaded erythrocyte has greater osmotic fragility than the unloaded one. Paired t-test is found, and the value suggests that the difference in osmotic fragility of both loaded and unloaded is not statistically significant. The osmotic fragility test of normal and loaded red blood cell is given in Fig. 10. 


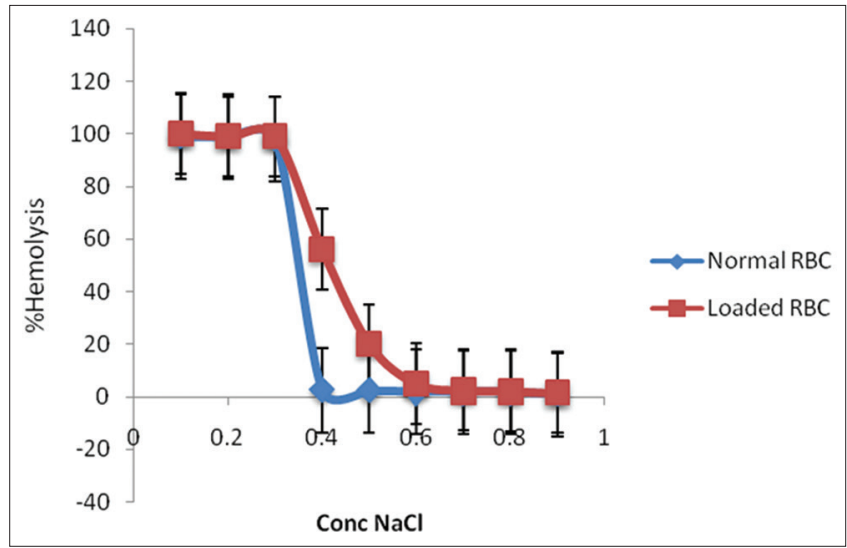

Fig. 10: The osmotic fragility test of normal and loaded red blood cell. \% hemolysis is ploted against concentration of sodium chloride. Data are expressed as mean \pm standard deviation, 3 samples in each group $n=3$

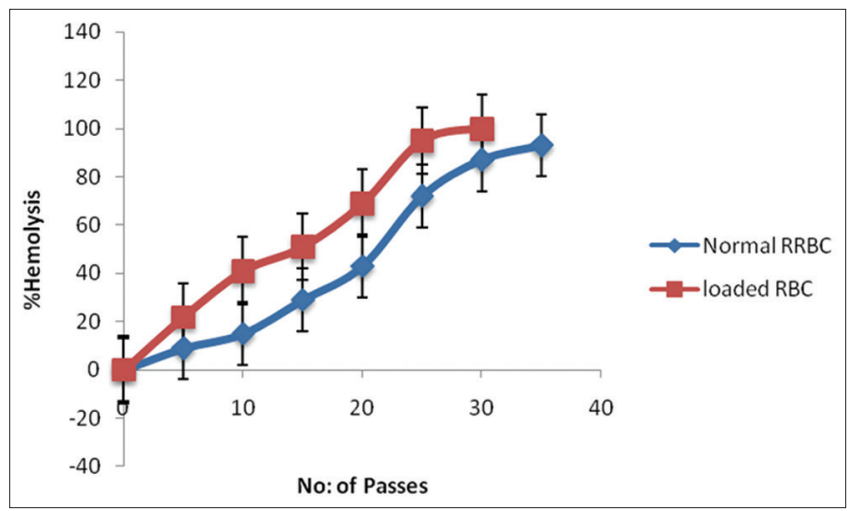

Fig. 11: The turbulence shock studies of normal and loaded red blood cell. \% hemolysis is ploted against number of passes through a syringe at a rate of $10 \mathrm{ml} /$ minutes. Data are expressed as mean \pm standard deviation, 3 samples in each group $n=3$

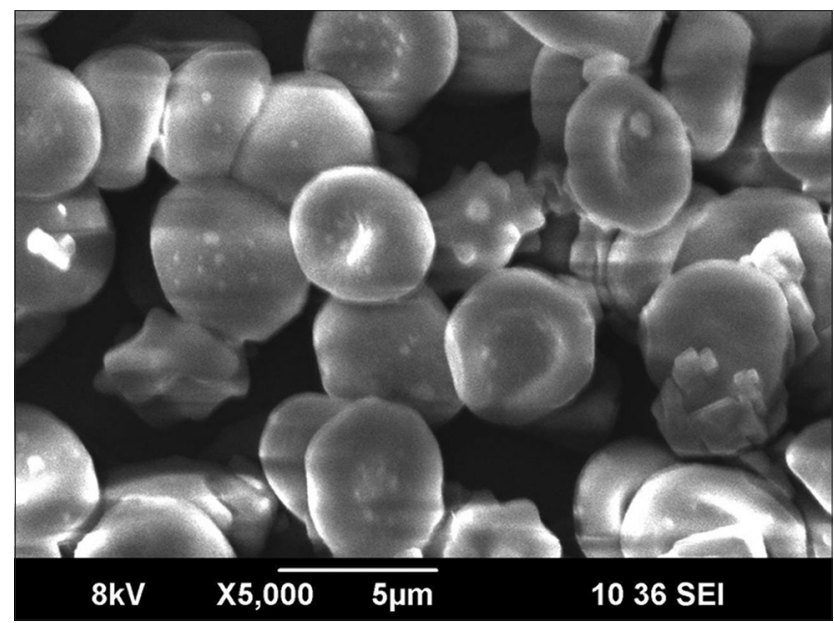

Fig. 12: Scanning electron micrograph image of normal erythrocytes

\section{Turbulence shock studies/turbulence fragility studies}

The disturbance easily-broken quality is one more (typical and expected) feature of erythrocytes that depends upon changes in the (honest and good human quality/wholeness or completeness) of cellular membrane and reflects resistance of loaded cells against blood destruction resulting from (full of violently swirling disorder) flow within circulation. Turbulence test provides the idea about the shock that is supposed to be affected to erythrocytes when it passes through small capillaries of human body. The turbulence shock was calculated for both loaded and unloaded erythrocytes and the significant difference between the loaded and normal erythrocytes and paired t-test value suggested even though the turbulence shock is greater for loaded erythrocytes, the difference is not considered to be statistically significant. The turbulance shock studies of normal and loaded red blood cells is given in Fig. 11.

\section{Cell counting and cell recovery}

$\%$ cell recovered was calculated and was found to be $73-78 \%$, which suggests that the loading technique was less destructive.

\section{Morphological studies}

To find out the morphological difference of erythrocyte after loading of metformin by endcytosis method, the SEM image of both loaded and normal was taken using JEOL JSM-6390 LA scanning electron microscope. The some echinocytes transition was seen even in normal RBC which might be due to storage lesion. But the echinocyte was significantly less in number compared to discocytes. The SEM image of normal RBC is given in Fig. 12.

Even after loading metformin also, there was no significant shape transformation had occurred. SEM study result suggests that biconcave shape of RBC is retained even after loading. This suggests that loading metformin by the process of endocytosis was a less destructive technique.

\section{Determination of phospholipid asymmetry}

Maintenance of phospholipid asymmetry of erythrocyte was checked using in order to determine whether the loading process or the drug metformin had any role in disturbing the lipid asymmetry of erythrocyte. Like, any other cells, erythrocyte is also composed of phospholipid bilayer. The outer layer of it is uncharged while inner layer is charged. The former is composed of PC $(30 \%)$ and SM $(25 \%)$ while latter is composed of lipids like phosphatidyl ethanolamine $(28 \%)$ and PS (14\%). Between the lipoid bilayer is the cholesterol. These phospholipids are non randomly arranged in bilayer in normal cells. Hence the name lipid asymmetry. Phospholipid asymmetry is maintained throughout the lifespan of the cell. Once the cell enters into apoptosis stage, this phospholipid asymmetry gets distorted. When lipid asymmetry is distorted, amine containing phospholipids (PS) will expose, PS act as a selfmarker for macrophages and removed from the body by engulfing them. Erythrocytes can be used as drug carrier for slow release depot formulation provided that there should not any exposure of PS during the loading procedure. Exposure of PS would lead to rapid recognition of erythrocytes by mononuclear phagocytic system. Detection of phospholipid asymmetry is studied by binding with fluorescently labeled Annexin V. Annexin V is a $35-36 \mathrm{kDa}$ Ca2+ dependent phospholipid-binding protein that has a high affinity for PS, and binds to cells with exposed PS.

Quadrant 3 (Q3): This quadrant shows the no: Of cells that do not have affinity for Annexin, which means all cells are live and no exposure of PS.

Quadrant 4 (Q4): Annexin V positive cell is quantified in this table. That means it gives information about the apoptotic cell or PS exposed erythrocytes.

Q1 and Q2 have no role in determining the phospholipid asymmetry of RBC as these quadrants are meant for nucleus-containing cells. This quadrants give positive results for propidium iodide dye which stains the nucleus. From the four quadrant table, it was observed that there was significantly less amount of cells in Q4 region for both loaded and normal erythrocyte. Hence it revealed that the loading of metformin in erythrocytes by the method of Endocytosis was a less destructive technique. The numbers of exposed PS cells were significantly less. 
Hence, the drug loaded erythrocyte would remain in the circulation without recognized by the mononuclear phagocytic system. That means the metformin loaded erythrocyte would remain in the circulation exactly the same life span of normal RBC.

\section{MCV}

The mean cell quantity points to shows the volume of the "average" red cell in a sample. MCV is higher or decreased (going along with) average red cell size; i.e., low MCV points to/shows microcytic (small average RBC size), (usual/commonly and regular/healthy) MCV points to normocytic ((usual average RBC size), and high MCV points to macrocytic (large average RBC size). The reference range for MCV is 80-96 fL/red cell in adult. The unloaded erythrocyte shows an MCV value of $93 \mathrm{fl}$ and metformin loaded erythrocytes shows an MCV value of $95 \mathrm{fl}$ indicates a normal average RBC size even after loading.

\section{Stability studies}

There is no prescribed stability studies guideline for resealed erythrocyte formulation. According to literature survey, resealed erythrocytes are lyophilized after loading and stored at $4^{\circ} \mathrm{C}$. However, the SEM image of lyophilized product results in the distortion of RBC shape. Researchers are going on in the field of freeze-drying of blood product; yet no successful outcome was reported.

From the table, it was revealed that for F0 formulation that is formulation with no glutaraldehyde cross-linking was stable only for 28 days at $4^{\circ} \mathrm{C}$ in erythrosol solution.

\section{CONCLUSION}

This study aimed to retard the release of metformin by encapsulating in carrier erythrocyte. The encapsulation was done by the method of endocytosis so that the metformin would reside inside the endocytic vacuoles. Hence, the release might be retarded for the metformin which was having a very small molecular weight. In addition, from the study, it was revealed that some amount of metformin encapsulated by the mechanism of diffusion also. Metformin had hydrogen bonding with hemoglobin as suggested by docking study. Both endocytosis and diffusion take place in drug loading mechanism. The loaded cells were then characterized in vitro for osmotic fragility, drug release, resistance to turbulence shock, mean corpuscular volume, and maintenance of phospholipid asymmetry. The cells showed higher osmotic easilybroken quality and lower resistance to disturbance shock as compared to the healthy cells. It was found about $98.34 \%$ of metformin release for about 12 days. A near zero order release kinetics was observed from metformin loaded erythrocytes and glutaraldehyde treatment of loaded cells resulted in further drug release retardation. Lipid asymmetry of erythrocyte was maintained even after loading metformin which suggests the metformin loaded erythrocyte would remain in the circulation with exactly the same half-life of normal erythrocyte. The near zero order release kinetics, comparable hematological indices with normal erythrocytes and maintenance of lipid asymmetry of erythrocytes makes them a perfect carrier for parenteral slow release depot formulation of metformin. The loaded erythrocyte has all hematological and another parameter almost similar to normal erythrocytes; so would remain in the circulation with almost the same half-life of normal erythrocyte and the retardation in metformin release would decrease the dosing frequency. Even though the stability was less for resealed erythrocyte formulation, it would act as a perfect carrier for metformin for retarding the release.

\section{REFERENCES}

1. Prakash J. Hypertension and chronic kidney disease. In: Rana DS, editor. Systemic Diseases and Renal Manifestation. New Delhi: Elsevier Publication; 2009. p. 72-81.

2. Tan QY, Li HD, Zhu RH, Zhang QZ, Zhang J, Peng WX. Tolerability and pharmacokinetics of ranolazine following single and multiple sustained-release doses in Chinese healthy adult volunteers: A randomized, open-label, Latin square design, phase I study. Am J Cardiovasc Drugs 2013;13(1):17-25.

3. Davidson MB, Peters AL. An overview of metformin in the treatment of Type 2 diabetes mellitus. Am J Med 1997;102(1):99-110.

4. Fang RH, Hu CM, Zhang L. Nanoparticles disguised as red blood cells to evade the immune system. Expert Opin Biol Ther 2012;12(4):385-9.

5. Ashutosh M. Resealed erythrocytes: An engineering approach for drug delivery and drug targeting. Int J Pharm Pharm Sci 2016;8(5):376-84.

6. Pragya, Vaibhav R. Resealed erythrocytes: A promising drug delivery. Int J Pharm Pharm Sci 2012;4(3):75-82.

7. Park KM, Mrsny RJ. Controlled drug delivery: Present and future. In: Controlled Drug Delivery: Designing Technologies for the Future. Series, 752. Washington, DC: American Chemical Society; 2000. p. 2-3.

8. Schriei SL, Bensch KG, Johnson M, Junga I. Energized endocytosis in human erythrocyte ghosts. J Clin Invest 1975;56(1):8-22.

9. Corey EJ, Czakó B, Kürti L. Molecules and Medicine. Hoboken, NJ: Wiley; 2012

10. Dowler BC, editor. Endocytosis: Structural Components, Functions and Pathways. Hauppauge, NY: Nova Science Publishers; 2010.

11. Patel PD, Dand N, Hirlekar RS, Kadam VJ. Drug loaded erythrocytes: As novel drug delivery system. Curr Pharm Des 2008;14(1):63-70.

12. Dodov MG, Simonoska M, Goracinova K. Formulation and characterization of topical liposome gel bearing lidocaine $\mathrm{HCl}$. Bull Chem Technol Maced 2005;24(1):59-65.

13. Nayak UY, Gopal S, Mutalik S, Ranjith AK, Reddy MS, Gupta P, et al. Glutaraldehyde cross-linked chitosan microspheres for controlled delivery of zidovudine. J Microencapsul 2009;26(3):214-22.

14. Karbiner MS, Sierra L, Minahk C, Fonio MC, Bruno MP, Jerez S. The role of oxidative stress in alterations of hematological parameters and inflammatory markers induced by early hypercholesterolemia. Life Sci 2013;93(15):503-8.

15. Vyas SP, Jain SK. Preparation and in vitro characterization of a magnetically responsive ibuprofen-loaded erythrocytes carrier. J Microencapsul 1994;11(1):19-29.

16. Hortolà P. SEM examination of human erythrocytes in uncoated bloodstains on stone: Use of conventional as environmental-like SEM in a soft biological tissue (and hard inorganic material). J Microsc 2005;218:94-103.

17. Larson MC, Woodliff JE, Hillery CA, Kearl TJ, Zhao M. Phosphatidylethanolamine is externalized at the surface of microparticles. Biochim Biophys Acta 2012;1821(12):1501-7. 